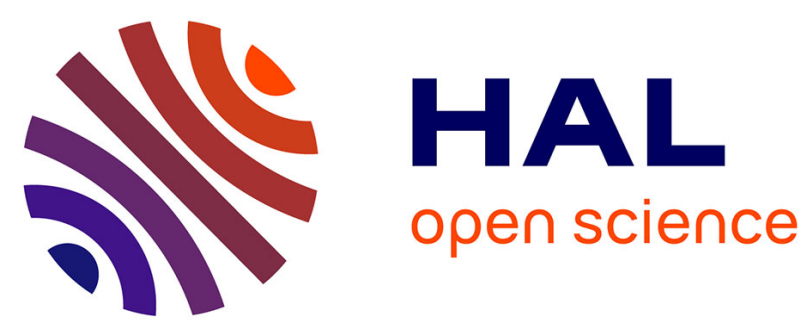

\title{
Importance Measure on a Finite Time Horizon and Application to Markovian Multi-State Production Systems
}

Phuc Do Van, Anne Barros, Christophe Bérenguer

\section{- To cite this version:}

Phuc Do Van, Anne Barros, Christophe Bérenguer. Importance Measure on a Finite Time Horizon and Application to Markovian Multi-State Production Systems. Proceedings of the Institution of Mechanical Engineers, Part O: Journal of Risk and Reliability, 2008, 222 (3), pp.449-461. 10.1243/1748006XJRR146 . hal-00705194

\section{HAL Id: hal-00705194 \\ https://hal.science/hal-00705194}

Submitted on 7 Jun 2012

HAL is a multi-disciplinary open access archive for the deposit and dissemination of scientific research documents, whether they are published or not. The documents may come from teaching and research institutions in France or abroad, or from public or private research centers.
L'archive ouverte pluridisciplinaire HAL, est destinée au dépôt et à la diffusion de documents scientifiques de niveau recherche, publiés ou non, émanant des établissements d'enseignement et de recherche français ou étrangers, des laboratoires publics ou privés. 


\title{
Importance Measure on a Finite Time Horizon and Application to Markovian Multi-State Production Systems
}

\author{
Phuc Do Van, Anne Barros, Christophe Bérenguer \\ Université de technologie de Troyes \\ Institut Charles Delaunay - FRE CNRS 2848 \\ Systems Modelling and Dependability Group \\ 12, rue Marie Curie - BP 2060 -10010 Troyes cedex - France \\ Tel : +33 325718027 - Fax: +33325715649 \\ Corresponding author: Christophe Bérenguer \\ E-mail : christophe.berenguer@utt.fr
}

\begin{abstract}
The sensitivity analysis of complex industrial systems aims at identifying, in a multi-unit structure, which components contribute the most to a variation of the performance criterion. In this paper an importance measure, called multi-directional sensitivity measure, defined as the derivative of the system performance in the direction defined by a vector (or matrix) in an appropriate space (e.g. direction of one parameter, of a group of parameters, or generally any direction of the transition rates of a Markov process) is considered. This measure proposed for sensitivity analysis of steady state reliability is developed herein for the transient state. It is also extended and applied to the study of the production capacity of multi-state production systems such as manufacturing, production lines, and power generation, which exhibit performances that can settle on different levels depending on the operative conditions of the constitutive components. A simple numerical example is introduced to show why this measure provides an efficient tool to investigate not only the importance of a given component, but also the importance of a class of components, the importance of the maintenance and, more generally, the effect of the simultaneous change of several
\end{abstract}


design parameters.

Keywords: reliability; sensitivity analysis; importance measure; Markov process; multi-state system; production system

\section{Introduction}

The reliability importance analysis of complex industrial systems aims at identifying, in a multiunit structure, which components contribute the most to a variation of the performance criterion. In classical reliability studies [15], many importance measures are considered (Birnbaum, FussellVesely, Critical importance measure, etc) to classify the different elements of a multi-unit system by order of importance. Hence, for example, the decisions for preventive and corrective maintenance, or the monitoring schedule, can be tuned as a function of this classification. Many studies have been done to improve the calculation of these measures, especially when the components can be considered as stochastically independent. In the realistic case of stochastic dependencies existing between some components (shared maintenance resource, cold spare, shared load, etc), the definition and the calculation of other criteria is needed and more and more different approaches are proposed. The exact solution for the sensitivity measures for a Markov model relies on the Frank's approach [6]: the classical set of differential equations is extended to a bigger set of equations including the sensitivity factor equations. However, this approach is computationally burdensome and almost unusable or highly inefficient on a realistic-size system when the state space dimension is too high. To cope with this problem, some approximate solutions have been proposed but they are often only applicable on a limited class of systems (e.g. acyclic Markov models with no repairs), [13]. Hence, the problem remains widely open.

For systems described by Markov Models, [5, 4] propose an importance measure, called multidirectional sensitivity measure (MSM), which corresponds to the derivative of the performance function (e.g. system availability or system production level) in the direction defined by a vector (or matrix) in an appropriate space (e.g. direction of one parameter, of a group of parameters, or generally any direction of the transition rates of a Markov process). In [5, 4], a procedure based on

perturbation analysis and one of its extensions presented in [2] is developed to evaluate MSM at steady state. The aim of the present paper is to show that the same importance measure MSM can be considered to conduct a reliability importance analysis in the transient state. Hence, the sen- 
sitivity analysis can be extended from steady state performances to transient state performances. This extension allows, for example, the sensitivity analysis of system performances on a finite time horizon. More precisely, this importance measure can provide an efficient tool to:

- identify the importance of a given component (parameter), and also the importance of a class of components with respect to the system performance of interest;

- evaluate the effect of the change in any direction of one parameter or a group of design parameters;

- solve maintenance policy optimization and performance improvement problems.

Moreover, from a practical point of view, many systems such as manufacturing production lines and power generation installations exhibit performances that can settle on different levels (e.g. $100 \%, 90 \%, 80 \%$ of the nominal capacity) depending on the operative conditions of the constitutive components. These components can be stochastically dependent [9] and the production capacity has often to be evaluated on a finite-time horizon, and not only at steady state. Many authors have defined importance measures for multi-state systems [10, 16, 14] but they mainly focus on universal generating function method and Monte Carlo simulation. In this paper, we also show that the multi-directional sensitivity measure MSM can be used to study the sensitivity of the production capacity in the context of Markovian multi-state production systems.

This paper is organized as follows. Section 2 is devoted to the presentation of MSM and the link with the classical importance measures, as well as the evaluation of MSM for a finite time horizon, and MSM for the average availability sensitivity during a given period of time. The link with MSM of the availability at steady-state, presented in [4], is also established. Section 3 focuses on the application to multi-state production systems. It is shown how MSM is used as an appropriate tool for the production capacity sensitivity analysis during a given time period of interest, as well as at steady-state. A simple numerical example is introduced in section 4 to illustrate the advantages of the proposed importance measure, MSM, for both reliability studies and production capacity sensitivity analysis. Finally, Section 5 presents the conclusions drawn from this work.

\section{Notation list}

M

$\mathbf{M}^{\sharp}$

Q transition rates matrix of Markov models

group inverse of $\mathbf{M}$

directional perturbation matrix/direction of sensitivity 


$\begin{array}{ll}\boldsymbol{P}(t) & \text { column vector of state probabilities at time } t \\ \tilde{\boldsymbol{P}}(t) & \text { column vector of average state probabilities vector during a given period time } \\ & {[0, t]} \\ \boldsymbol{\pi} & \text { column vector of steady-state probabilities } \\ A(t) & \text { system availability at time } t \\ \bar{A}(t) & \text { average availability during a given period time }[0, t] \\ A & \text { system availability at infinite time } \\ \boldsymbol{X} & \text { row vector of state production capacities } \\ S(t) & \text { expected production capacity at time } t \\ \bar{S}(t) & \text { average of expected production capacity during a given period time }[0, t] \\ S & \text { expected production capacity at infinite time } \\ \lambda & \text { failure rate of one unit } \\ \mu & \text { repair rate of one unit } \\ \text { MSM }_{\mathbf{D}}^{W} & \text { sensitivity of performance measure } W \text { in the direction } \mathbf{D}, \text { in which } W \text { and } \mathbf{D} \text { are } \\ & \text { either } A, S, \text { and } \lambda, \mathbf{Q}, \text { respectively }\end{array}$

\section{Importance measure on a finite time horizon}

Markov models are frequently used in reliability analysis to assess different metrics of interest, e.g. system reliability, availability, maintainability. Within this Markov modelling framework, the traditional reliability importance measures (e.g. Birnbaum importance or critical importance measure) used to analyze the system performance sensitivity with respect to the parameters of its components can be computationally expensive to evaluate. Moreover, in the context of more "complex" dynamic systems with inter-component and functional dependencies (cold spare, shared load, shared resources, etc), even the meaning and the definition of these traditional importance measures may be questionable. This section explores the multi-directional sensitivity measure MSM in reliability studies of Markovian systems on a finite time horizon (transient state), as well as a link with MSM at steady state presented in $[4,5]$.

\subsection{The multi-directional sensitivity measure}

Consider a dynamic system described by a Markov model and let the column vector $\boldsymbol{P}(t)$ be the vector of state probabilities, and $\boldsymbol{P}_{0}$ be the initial state probabilities vector. The system of the first order Chapman-Kolmogorov equations applied to homogeneous Markovian process (without 
additional dynamical variables) is

$$
\frac{d \boldsymbol{P}(t)}{d t}=\mathbf{M} \boldsymbol{P}(t)
$$

The solution of (1) can be expressed as

$$
\boldsymbol{P}(t)=e^{\mathbf{M} t} \boldsymbol{P}_{0}=F_{\mathbf{M}}(t) \boldsymbol{P}_{0}
$$

where $F_{\mathbf{M}}(t)=e^{\mathbf{M} t}$ is the exponential matrix.

A perturbation on one parameter or a group of parameters of the system is equivalent to a perturbation in the transition rates matrix M. It leads to a perturbed transition matrix

$$
\mathbf{M}_{\delta}=\mathbf{M}+\delta \mathbf{Q}
$$

where $\delta$ is a small real number and $\mathbf{Q}$ is a directional perturbation matrix in which an entry $Q_{i j}=0$ indicates that the transition rate from state $i$ to state $j$ is not perturbed and $Q_{i j}=\alpha$ different from 0 indicates that the transition rate from state $i$ to state $j$ is perturbed by an amount $\alpha \delta$. The only condition on the structure of $\mathbf{Q}$ to ensure that the matrix $\mathbf{M}_{\delta}$ remains a transition matrix is that the sum of each column of $\mathbf{Q}$ equals 0 .

Example: consider a system consisting of two independent units $C_{1}$ and $C_{2}$ in a parallel structure with constant failure rates $\lambda_{1}, \lambda_{2}$ and constant repair rates $\mu_{1}, \mu_{2}$, see Figure 1 . The transition matrix of this system is given by

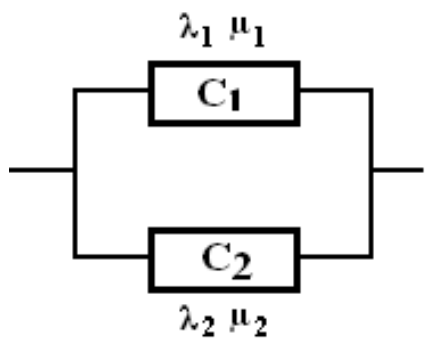

Figure 1: A parallel structure of 2 independent units

$$
\mathbf{M}=\left(\begin{array}{cccc}
-\lambda_{1}-\lambda_{2} & \mu_{1} & \mu_{2} & 0 \\
\lambda_{1} & -\mu_{1}-\lambda_{2} & 0 & \mu_{2} \\
\lambda_{2} & 0 & -\mu_{2}-\lambda_{1} & \mu_{1} \\
0 & \lambda_{2} & \lambda_{1} & -\mu_{1}-\mu_{2}
\end{array}\right)
$$

The state diagram of this system is sketched in Figures 2 and 3 for two different types of perturbations. Figure 2 sketches the Markov graph with a perturbation on one specific parameter, 


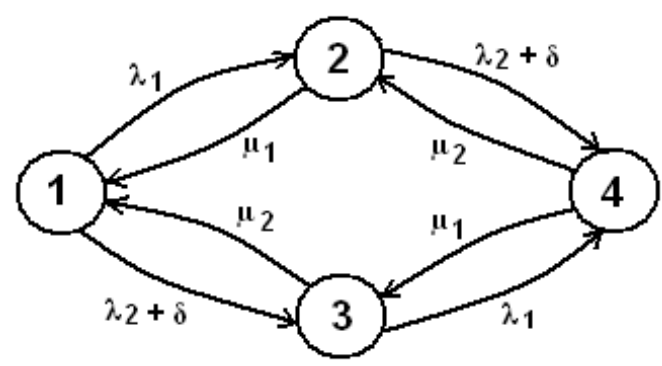

Figure 2: Perturbation on $\lambda_{2}$

State $1: C_{1} C_{2} \quad$ State $2: C_{1} \bar{C}_{2}$

State $3: \overline{C_{1}} C_{2} \quad$ State $4: \overline{C_{1}} \overline{C_{2}}$

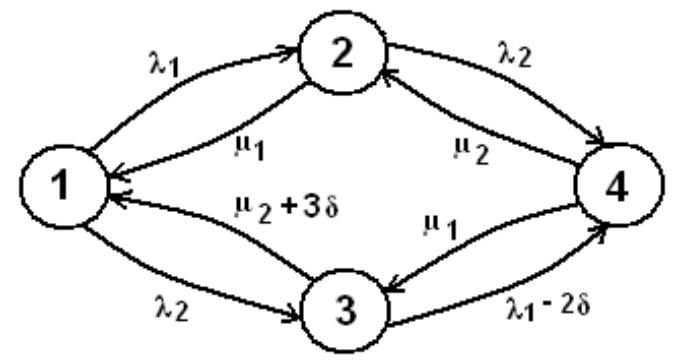

Figure 3: Perturbation on the exit transition rates of state 3

namely $\lambda_{2}$, which corresponds to the directional perturbation matrix $\mathbf{Q}_{1}$

$$
\mathbf{Q}_{1}=\left(\begin{array}{cccc}
-1 & 0 & 0 & 0 \\
0 & -1 & 0 & 0 \\
1 & 0 & 0 & 0 \\
0 & 1 & 0 & 0
\end{array}\right)
$$

Figure 3 presents the state diagram modified by a perturbation on the exit transition rates of one specific state, namely state number 3 . This perturbation corresponds to the directional perturbation matrix $\mathbf{Q}_{2}$

$$
\mathbf{Q}_{2}=\left(\begin{array}{cccc}
0 & 0 & 3 & 0 \\
0 & 0 & 0 & 0 \\
0 & 0 & -1 & 0 \\
0 & 0 & -2 & 0
\end{array}\right)
$$

The variations in the transition rates matrix affect the transient solution $\boldsymbol{P}(t)$ that becomes 
$\boldsymbol{P}_{\delta}(t)$ (with the same initial condition $\boldsymbol{P}_{\delta}(0)=\boldsymbol{P}(0)$ ). $\boldsymbol{P}_{\delta}(t)$ satisfies

$$
\frac{d \boldsymbol{P}_{\delta}(t)}{d t}=\mathbf{M}_{\delta} \boldsymbol{P}_{\delta}(t)
$$

The derivative of $\boldsymbol{P}(t)$ in the direction of $\mathbf{Q}$ is defined as the following

$$
\frac{d \boldsymbol{P}(t)}{d \mathbf{Q}}=\lim _{\delta \rightarrow 0} \frac{\boldsymbol{P}_{\delta}(t)-\boldsymbol{P}(t)}{\delta}
$$

The system availability at time $t$ is defined as

$$
A(t)=\sum_{i \in \Omega_{O}} \boldsymbol{P}_{i}(t)=\boldsymbol{f} \boldsymbol{P}(t)
$$

where $\Omega_{O}$ is, for binary systems, a set of operational states, and $\boldsymbol{f}=\left(f_{1}, f_{2}, \ldots, f_{n}\right)$ is a row vector associated with the system states, e.g. if $f_{i}=1$ if system is in operational state $i$ and $f_{i}=0$ otherwise. For multi-state systems, $\Omega_{O}$ is a set of states whose level of performance are higher than the level required (see e.g., $[10,16])$.

The sensitivity of $A(t)$ in the direction $\mathbf{Q}$, named $\operatorname{MSM}_{\mathbf{Q}}^{A}(t)$, is defined as

$$
\operatorname{MSM}_{\mathbf{Q}}^{A}(t)=\frac{d A(t)}{d \mathbf{Q}}=\boldsymbol{f} \frac{d \boldsymbol{P}(t)}{d \mathbf{Q}}
$$

It is clear that $\operatorname{MSM}_{\mathbf{Q}}^{A}(t)$ represents the sensitivity of the system availability in the direction of interest $\mathbf{Q}$ that corresponds to the direction of one parameter, the direction of a group of parameters (failure rates, repair rates for example), or more generally, the direction of a group of transition rates.

Link with classical importance measure Looking at the example shown in Figure 1, since $\mathbf{Q}_{1}$ indicates the perturbation of only one parameter of the system $\left(\lambda_{2}\right), \operatorname{MSM}_{\mathbf{Q}_{1}}^{A}(t)$ corresponds therefore to $\partial A / \partial \lambda_{2}$, i.e. the partial derivative of the system availability with respect to $\lambda_{2}$. In this case, the link with the classical Birnbaum's importance measure [15], defined as the derivative of the system availability with respect to the availability of a given component in the context of independent components system, is directly established using the chain rule

$$
I^{B}\left(C_{2} / t\right)=\frac{\partial A(t)}{\partial a_{2}(t)}=\frac{\partial A(t)}{\partial \lambda_{2}} / \frac{\partial a_{2}(t)}{\partial \lambda_{2}}
$$


or,

$$
I^{B}\left(C_{2} / t\right)=\operatorname{MSM}_{\mathbf{Q}_{1}}^{A}(t) / \frac{\partial a_{2}(t)}{\partial \lambda_{2}}
$$

where $a_{2}(t)$ is the availability of component 2 .

Note however that if there exist dependencies between the components, e.g. if in a two-unit system $C_{2}$ is in cold redundancy with $C_{1}$ (see Figure 4) the definition of an importance measure as the partial derivative of the system availability with respect to a component availability may be questionable. Indeed, the availability of component does not depend only of its characteristics but

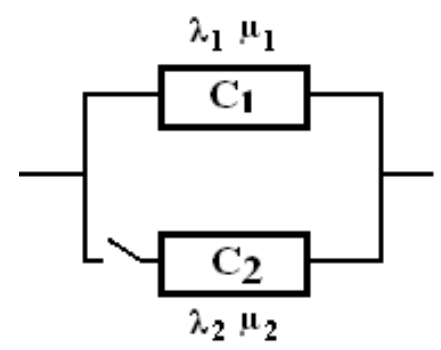

Figure 4: Cold redundancy structure

also on other system parameters, and its availability in the system can be different of its availability out of the system [13]. In this context, the partial derivative with respect to a parameter, rather than to the availability of a component, appears to be more relevant and is often preferred for design purpose. Hence, the multi-directional sensitivity measure MSM proposed in this work offers an interesting (and generalizing) alternative, especially for dynamic systems with inter-components dependencies. More precisely, it can provide an efficient tool to

- identify the importance of a given component (parameter), and also the importance of a class of components;

- evaluate the effect of the change in any direction of one or more design parameters;

- optimize the maintenance policy problems, reliability improvement problems.

\subsection{Evaluation of MSM}

\subsubsection{Transient state}

Let $\boldsymbol{Z}(t)=\boldsymbol{P}_{\delta}(t)-\boldsymbol{P}(t)$. From Equations (1) and (3), $\boldsymbol{Z}(t)$ satisfies

$$
\frac{d \boldsymbol{Z}(t)}{d t}=\mathbf{M}_{\delta} \boldsymbol{Z}(t)+\left(\mathbf{M}_{\delta}-\mathbf{M}\right) \boldsymbol{P}(t)
$$


given the initial condition $\boldsymbol{Z}(0)=\boldsymbol{P}_{\delta}(0)-\boldsymbol{P}(0)=\mathbf{0}$. The solution to the nonhomogeneous linear equation (6) can be expressed as (see, e.g., [7] for the details)

$$
\boldsymbol{Z}(t)=\int_{0}^{t} e^{\mathbf{M}_{\delta}(t-s)}\left(\mathbf{M}_{\delta}-\mathbf{M}\right) \boldsymbol{P}(s) d s
$$

or,

$$
\boldsymbol{Z}(t)=\int_{0}^{t} F_{\mathbf{M}_{\delta}}(t-s)\left(\mathbf{M}_{\delta}-\mathbf{M}\right) \boldsymbol{P}(s) d s
$$

Replacing $\boldsymbol{P}_{\delta}(t)-\boldsymbol{P}(t)$ in (4) by $\boldsymbol{Z}(t)$ and using (7), the derivative of $\boldsymbol{P}(t)$ in the direction $\mathbf{Q}$ can be expressed as

$$
\begin{gathered}
\frac{d \boldsymbol{P}(t)}{d \mathbf{Q}}=\lim _{\delta \rightarrow 0} \frac{1}{\delta} \int_{0}^{t} F_{\mathbf{M}_{\delta}}(t-s)\left(\mathbf{M}_{\delta}-\mathbf{M}\right) \boldsymbol{P}(s) d s . \\
\frac{d \boldsymbol{P}(t)}{d \mathbf{Q}}=\lim _{\delta \rightarrow 0} \int_{0}^{t} F_{\mathbf{M}_{\delta}}(t-s) \frac{\mathbf{M}_{\delta}-\mathbf{M}}{\delta} \boldsymbol{P}(s) d s .
\end{gathered}
$$

Using $\mathbf{Q}=\left(\mathbf{M}_{\delta}-\mathbf{M}\right) / \delta$

$$
\begin{gathered}
\frac{d \boldsymbol{P}(t)}{d \mathbf{Q}}=\int_{0}^{t}\left(\lim _{\delta \rightarrow 0} F_{\mathbf{M}_{\delta}}(t-s)\right) \mathbf{Q P}(s) d s . \\
\frac{d \boldsymbol{P}(t)}{d \mathbf{Q}}=\int_{0}^{t} F_{\mathbf{M}}(t-s) \mathbf{Q P}(s) d s .
\end{gathered}
$$

Substituting (2) in to (8) we obtain

$$
\frac{d \boldsymbol{P}(t)}{d \mathbf{Q}}=\int_{0}^{t} F_{\mathbf{M}}(t-s) \mathbf{Q} F_{\mathbf{M}}(s) \boldsymbol{P}_{0} d s
$$

Finally, from the definition (5), the MSM of $A(t)$ in the direction $\mathbf{Q}$ can be written as

$$
\operatorname{MSM}_{\mathbf{Q}}^{A}(t)=\int_{0}^{t} \boldsymbol{f} F_{\mathbf{M}}(t-s) \mathbf{Q} F_{\mathbf{M}}(s) \boldsymbol{P}_{0} d s
$$

This may be evaluated by a numerical integration method or directly by making a suitable expansion of matrix exponentials with, for example, the uniformization method [12].

Equation (10) allows for the evaluation of the system availability sensitivity in any direction of interest at time $t$ in the transient state. A similar formula evaluation has been shown in [1] by using the generalized perturbation theory (GPT) method in the context of Makovian systems. However, the direction $\mathbf{Q}$ in [1] is limited to the direction of a single parameter. 


\subsubsection{Average on a finite time horizon}

From (6) by taking integrals for a given period of time $[0, t]$ we obtain the following differential equation

$$
\int_{0}^{t} \frac{d \boldsymbol{Z}(s)}{d s} d s=\mathbf{M}_{\delta} \int_{0}^{t} \boldsymbol{Z}(s) d s+\left(\mathbf{M}_{\delta}-\mathbf{M}\right) \int_{0}^{t} \boldsymbol{P}(s) d s
$$

or,

$$
\boldsymbol{Z}(t)-\boldsymbol{Z}(0)=\mathbf{M}_{\delta} \int_{0}^{t} \boldsymbol{Z}(s) d s+\left(\mathbf{M}_{\delta}-\mathbf{M}\right) \int_{0}^{t} \boldsymbol{P}(s) d s
$$

Let us define:

- $\tilde{\boldsymbol{P}}(t)=\int_{0}^{t} \boldsymbol{P}(s) d s$

- $\tilde{\boldsymbol{Z}}(t)=\tilde{\boldsymbol{P}}_{\delta}(t)-\tilde{\boldsymbol{P}}(t)=\int_{0}^{t} \boldsymbol{Z}(s) d s$.

Note that $d \tilde{\boldsymbol{Z}}(t) / d t=\boldsymbol{Z}(t)$ and $\boldsymbol{Z}(0)=\mathbf{0}$, the differential equation (11) can therefore be written as

$$
\frac{d \tilde{\boldsymbol{Z}}(t)}{d t}=\mathbf{M}_{\delta} \tilde{\boldsymbol{Z}}(t)+\left(\mathbf{M}_{\delta}-\mathbf{M}\right) \tilde{\boldsymbol{P}}(t)
$$

whose the solution is

$$
\tilde{\boldsymbol{Z}}(t)=\int_{0}^{t} F_{\mathbf{M}_{\delta}}(t-s)\left(\mathbf{M}_{\delta}-\mathbf{M}\right) \tilde{\boldsymbol{P}}(s) d s
$$

The derivative of $\tilde{\boldsymbol{P}}(t)$ in the direction $\mathbf{Q}$ is expressed as

$$
\frac{d \tilde{\boldsymbol{P}}(t)}{d \mathbf{Q}}=\lim _{\delta \rightarrow 0} \frac{\tilde{\boldsymbol{Z}}(t)}{\delta}=\lim _{\delta \rightarrow 0} \int_{0}^{t} F_{\mathbf{M}_{\delta}}(t-s) \mathbf{Q} \tilde{\boldsymbol{P}}(s) d s,
$$

or,

$$
\frac{d \tilde{\boldsymbol{P}}(t)}{d \mathbf{Q}}=\int_{0}^{t} F_{\mathbf{M}}(t-s) \mathbf{Q} \tilde{\boldsymbol{P}}(s) d s .
$$

The average availability during a given period $[0, t]$

$$
\bar{A}(t)=\frac{1}{t} \int_{0}^{t} A(s) d s=\frac{1}{t} \int_{0}^{t} \boldsymbol{f} \boldsymbol{P}(s) d s=\frac{1}{t} \boldsymbol{f} \tilde{\boldsymbol{P}}(t) .
$$

The sensitivity of $\bar{A}(t)$ in the direction $\mathbf{Q}$ (i.e. the MSM of $\bar{A}(t))$ is finally

$$
\operatorname{MSM}_{\mathbf{Q}}^{\bar{A}}=\frac{d \bar{A}(t)}{d \mathbf{Q}}=\frac{1}{t} f \frac{d \tilde{\boldsymbol{P}}(t)}{d \mathbf{Q}}=\frac{1}{t} \boldsymbol{f} \int_{0}^{t} F_{\mathbf{M}}(t-s) \mathbf{Q} \tilde{\boldsymbol{P}}(s) d s .
$$

This equation allows the calculation of the average availability sensitivity during a given period $[0, t]$ in any direction of interest $\mathbf{Q}$. 


\subsubsection{Link with the steady-state}

If the Markov process is irreducible then when $t$ tends towards infinity, the system reaches a steady state behavior, so $\lim _{t \rightarrow \infty}\{d A(t) / d t\}=0$. Let $\boldsymbol{\pi}=\left(\pi_{1}, \pi_{2}, \ldots \pi_{n}\right)^{\prime}$ be the column vector representing the steady state probabilities $\left(\boldsymbol{\pi}=\lim _{t \rightarrow \infty} \boldsymbol{P}(t)\right)$, and let $\boldsymbol{Z}_{\boldsymbol{\pi}}=\lim _{t \rightarrow \infty} \boldsymbol{Z}(t)$, then Equation (6) becomes

$$
\mathbf{M}_{\delta} \boldsymbol{Z}_{\boldsymbol{\pi}}+\left(\mathbf{M}_{\delta}-\mathbf{M}\right) \boldsymbol{\pi}=0
$$

or

$$
-\mathbf{M}_{\delta} \frac{Z_{\boldsymbol{\pi}}}{\delta}=\mathbf{Q} \boldsymbol{\pi}
$$

Since matrix $\mathbf{M}_{\delta}$ is not invertible, the generalized inverse (or group inverse) $\mathbf{M}_{\delta}^{\sharp}=\left(\mathbf{M}_{\delta}-\boldsymbol{\pi}_{\delta} \boldsymbol{e}\right)^{-1}-$ $\boldsymbol{\pi}_{\delta} \boldsymbol{e}$, with $\boldsymbol{e}=(1,1 \ldots)$, has to be used to solve Equation (15) for $\boldsymbol{Z}_{\boldsymbol{\pi}}$, see [11] for details. Using the relations $\mathbf{M}_{\delta}^{\sharp} \mathbf{M}_{\delta}=\mathbf{I}-\boldsymbol{\pi}_{\delta} \boldsymbol{e}$ and $\boldsymbol{e} \boldsymbol{\pi}=\boldsymbol{e} \boldsymbol{\pi}_{\delta}=1$, it follows that

$$
\frac{Z_{\boldsymbol{\pi}}}{\delta}=-\mathbf{M}_{\delta}^{\sharp} \mathbf{Q} \boldsymbol{\pi} .
$$

The derivative of $\boldsymbol{\pi}$ in the direction of $\mathbf{Q}$ can be defined as

$$
\frac{d \boldsymbol{\pi}}{d \mathbf{Q}}=\lim _{\delta \rightarrow 0} \frac{\boldsymbol{Z}_{\boldsymbol{\pi}}}{\delta}=-\lim _{\delta \rightarrow 0} \mathbf{M}_{\delta}^{\sharp} \mathbf{Q} \boldsymbol{\pi} .
$$

Since $\mathbf{M}_{\delta}^{\sharp}$ is continuous, i.e., $\lim _{\delta \rightarrow 0} \mathbf{M}_{\delta}^{\sharp}=\mathbf{M}^{\sharp}=(\mathbf{M}-\boldsymbol{\pi} \boldsymbol{e})^{-1}-\boldsymbol{\pi} \boldsymbol{e}[2], d \boldsymbol{\pi} / d \mathbf{Q}$ can be expressed as

$$
\frac{d \boldsymbol{\pi}}{d \mathbf{Q}}=-\mathbf{M}^{\sharp} \mathbf{Q} \boldsymbol{\pi} .
$$

Let us note $A=\lim _{t \rightarrow \infty} A(t)=\boldsymbol{f} \boldsymbol{\pi}$, the system availability at infinite time (steady state). Hence the sensitivity of $A$ in the direction $\mathbf{Q}$ (i.e. the MSM of $A$ ) can be written as

$$
\mathbf{M S M}_{\mathbf{Q}}^{A}=\frac{d A}{d \mathbf{Q}}=-\boldsymbol{f} \mathbf{M}^{\sharp} \mathbf{Q} \boldsymbol{\pi} .
$$

The exact solution is obtained by calculating the group inverse [11]. An estimate solution has been proposed by Cao in [2]: $\boldsymbol{G}=\boldsymbol{f} \mathbf{M}^{\sharp}$, called potential vector, can be estimated directly from a single sample path observation. This method seems to be very powerful for Markov sensitivity analysis and Markov decision-making problems and it is used to study the reliability sensitivity analysis for steady-state systems in [4]. 


\section{Application to multi-state production systems}

For multi-state production systems such as e.g. manufacturing production lines and power generation installations, the performances output of interest is not only the reliability but also the production capacity $[9,8]$. This section explores, in the framework of Makovian multi-state production systems, how MSM can be extended to assess the sensitivity of production capacity with respect to the reliability characteristics of the system.

Assume that a unique production (or treatment) capacity $X_{i}$ corresponds to each state $i$ and let $P_{i}(t)$ be the probability of being in state $i$ at time $t$. The expected production capacity at time $t$ is then

$$
S(t)=\mathbb{E}_{\boldsymbol{P}(t)}\{\boldsymbol{X}\}=\sum_{i \in \Omega} P_{i}(t) X_{i}=\boldsymbol{X} \boldsymbol{P}(t)
$$

where $\boldsymbol{X}=\left(X_{1}, X_{2}, \ldots, X_{m}\right)$ is a row vector representing the state production capacities, $\mathbb{E}_{\boldsymbol{P}}(t)$ devotes the expectation with respect to the state probabilities $\boldsymbol{P}(t)$, and $\Omega$ is the state space of the production system.

Note well that $X_{i}$ depend on the production capacity of the components and on the system structure. To evaluate the production capacities vecteur $\boldsymbol{X}$, we consider that components are defined as the level at which the failure and repair rate data are collected or estimated. Relevant states, like functioning, failed or degraded, should be defined for each component. The production capacity of each system state is calculated by splitting the system into subsystems and basic subsystems which are series or parallel structures.

Let $X_{k}^{\text {struct. }}$ represent the production capacity of a structure (subsystem or basic subsystem) with $n$ units that is in state $k(k=1,2, \ldots)$. Let $Y_{i}^{k}$ represent the production capacity level of component $i$ when the system (or the structure) is in a state $k$. One gets

- for a series structure

$$
X_{k}^{\text {series-struct. }}=\min \left(Y_{1}^{k}, Y_{2}^{k}, \ldots, Y_{n}^{k}\right)
$$

- for a parallel structure

$$
X_{k}^{\text {parallel-struct. }}=\sum_{i=1}^{n} Y_{i}^{k} .
$$

Using (9) and (18), the directional sensitivity (or directional derivative) of the expected production capacity in the direction $\mathbf{Q}$ (i.e. the MSM of $S(t)$ ) at time $t$ is written as

$$
\operatorname{MSM}_{\mathbf{Q}}^{S}(t)=\frac{d S(t)}{d \mathbf{Q}}=\boldsymbol{X} \int_{0}^{t} F_{\mathbf{M}}(t-s) \mathbf{Q} F_{\mathbf{M}}(s) \boldsymbol{P}_{0} d s
$$


The average production capacity during a given period $[0, t]$ is defined as

$$
\bar{S}(t)=\frac{1}{t} \int_{0}^{t} \boldsymbol{X} \boldsymbol{P}(s) d s
$$

or,

$$
\bar{S}(t)=\frac{\boldsymbol{X}}{t} \tilde{\boldsymbol{P}}(t)
$$

Using (13) and (20), the sensitivity of the average production capacity during a given period in the direction of interest $\mathbf{Q}$ (i.e. the MSM of $\bar{S}(t)$ ) can be expressed as

$$
\operatorname{MSM}_{\mathbf{Q}}^{\bar{S}}=\frac{d \bar{S}(t)}{d \mathbf{Q}}=\frac{\boldsymbol{X}}{t} \int_{0}^{t} F_{\mathbf{M}}(t-s) \mathbf{Q} \tilde{\boldsymbol{P}}(s) d s
$$

If the system reaches a steady state (case of irreducible Markov process), then when $t$ tends towards infinity, $\lim _{t \rightarrow \infty} \bar{S}(t)=\lim _{t \rightarrow \infty} S(t)=\boldsymbol{X} \boldsymbol{\pi}$. Let $S=\boldsymbol{X} \boldsymbol{\pi}, S$ is called the production capacity at steady state. So using (16), the sensitivity of the production capacity at steady-state in the direction $\mathbf{Q}$ (i.e. the MSM of $S$ ) can be written as

$$
\operatorname{MSM}_{\mathbf{Q}}^{S}=\frac{d S}{d \mathbf{Q}}=\boldsymbol{X} \frac{d \boldsymbol{\pi}}{d \mathbf{Q}}=-\boldsymbol{X} \mathbf{M}^{\sharp} \mathbf{Q} \boldsymbol{\pi} .
$$

The multi-directional sensitivity measure MSM can be used in multi-state production systems to evaluate the variation of production capacity at time $t$ (or for a given period) when one or a group of parameters changes their value at the same time. It is also useful to find the importance of one parameter/component or even of a group of parameters/components for the system production capacity.

\section{Numerical example}

The purpose of this section is to show how the MSM can be used in reliability importance analysis and in production capacity analysis through a simple example. Both availability and production capacity criteria are considered for the transient state of a Markovian system, for its steady state and also for a given time period of interest.

Figure 5 represents a part of a production line with 4 units divided into 2 groups

- Group G1: units $C_{1}$ and $C_{2}$ are treatment units. Their production capacities are 100 products/hour (hr) (for normal operation state), and 0 (for failed state). When $C_{2}$ fails, the production capacity of unit $C_{1}$ increases by $20 \%$ (for simplicity, all capacities can be nor- 


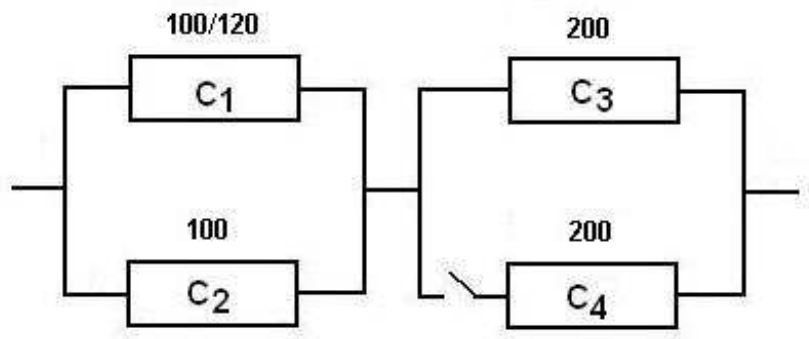

Figure 5: A part of production line.

Table 1: System states

\begin{tabular}{|c|c|c|c|c|c|c|}
\hline \multirow[b]{2}{*}{ State } & \multicolumn{4}{|c|}{ Component } & \multirow[b]{2}{*}{ System } & \multirow[b]{2}{*}{ production capacity } \\
\hline & $C_{1}$ & $C_{2}$ & $C_{3}$ & $C_{4}$ & & \\
\hline 1 & $\mathrm{O}$ & $\mathrm{O}$ & $\mathrm{O}$ & S & $\mathrm{O}$ & 200 \\
\hline 2 & $\mathrm{~F}$ & $\mathrm{O}$ & $\mathrm{O}$ & S & $\mathrm{O}$ & 10 \\
\hline 3 & $\mathrm{O}$ & $\mathrm{F}$ & $\mathrm{O}$ & S & $\mathrm{O}$ & 120 \\
\hline 4 & $\mathrm{O}$ & $\mathrm{O}$ & $\mathrm{F}$ & $\mathrm{O}$ & $\mathrm{O}$ & 200 \\
\hline 5 & $\mathrm{O}$ & $\mathrm{O}$ & $\mathrm{O}$ & $\mathrm{F}$ & $\mathrm{O}$ & 200 \\
\hline 6 & $\mathrm{~F}$ & $\mathrm{~F}$ & $\mathrm{O}$ & S & $\mathrm{F}$ & 0 \\
\hline 7 & $\mathrm{~F}$ & $\mathrm{O}$ & $\mathrm{F}$ & $\mathrm{O}$ & $\mathrm{O}$ & 100 \\
\hline 8 & $\mathrm{~F}$ & $\mathrm{O}$ & $\mathrm{O}$ & $\mathrm{F}$ & $\mathrm{O}$ & 100 \\
\hline 9 & $\mathrm{O}$ & $\mathrm{F}$ & $\mathrm{F}$ & $\mathrm{O}$ & $\mathrm{O}$ & 120 \\
\hline 10 & $\mathrm{O}$ & $\mathrm{F}$ & $\mathrm{O}$ & $\mathrm{F}$ & $\mathrm{O}$ & 120 \\
\hline 11 & $\mathrm{O}$ & $\mathrm{O}$ & $\mathrm{F}$ & $\mathrm{F}$ & $\mathrm{F}$ & 0 \\
\hline 12 & $\mathrm{~F}$ & $\mathrm{~F}$ & $\mathrm{~F}$ & $\mathrm{O}$ & $\mathrm{F}$ & 0 \\
\hline 13 & $\mathrm{~F}$ & $\mathrm{~F}$ & $\mathrm{O}$ & $\mathrm{F}$ & $\mathrm{F}$ & 0 \\
\hline 14 & $\mathrm{~F}$ & $\mathrm{O}$ & $\mathrm{F}$ & $\mathrm{F}$ & $\mathrm{F}$ & 0 \\
\hline 15 & $\mathrm{O}$ & $\mathrm{F}$ & $\mathrm{F}$ & $\mathrm{F}$ & $\mathrm{F}$ & 0 \\
\hline
\end{tabular}

malized, and they actually represent a given amount of products/hr)

- Group G2: units $C_{3}$ and $C_{4}$ are packaging units, and $C_{4}$ is in cold redundancy with $C_{3}$. $C_{3}$ is the main operating unit of group $\mathrm{G} 2$ : as soon as $C_{3}$ is repaired, $C_{4}$ stops and priority is always given to the repair of $C_{3}$. The production capacity values of $C_{3}$ and $C_{4}$ are 200 products/hr and 0 corresponding to the running state and the failed state respectively (there are no degraded condition for them).

The nominal production capacity of the system is 200 products/hr. The operational mode of the system is described in Table 1 where "O" denotes an operating state, " $\mathrm{S}$ " denotes a standby state, and "F" denotes a failed state. The corresponding Markov process is sketched in Figure 6. Table 2 gives the values of failure rates $\lambda_{i}$ ( $\bar{\lambda}_{i}$ for failure of shared load case, when $C_{2}$ fails and $C_{1}$ is functioning, for example), the repair rates $\mu_{i},(i=1, \ldots, 4)$, as well as the production capacities of each component. 
Table 2: Transition rates \& production capacities.

\begin{tabular}{ccccc}
\hline Units & $\lambda_{i}$ & $\mu_{i}$ & $\bar{\lambda}_{i}$ & Production capacity \\
\hline$C_{1}$ & $4.5 \mathrm{e}-4$ & $4 \mathrm{e}-3$ & $1 \mathrm{e}-3$ & $0 / 100 / 120$ \\
$C_{2}$ & $4.5 \mathrm{e}-4$ & $4 \mathrm{e}-3$ & - & $0 / 100$ \\
$C_{3}$ & $4.5 \mathrm{e}-4$ & $3 \mathrm{e}-3$ & - & $0 / 200$ \\
$C_{4}$ & $6.0 \mathrm{e}-4$ & $3 \mathrm{e}-3$ & - & $0 / 200$ \\
\hline
\end{tabular}



Figure 6: Markov diagram \& production capacities distribution.

Consider first the system availability $A(t)$ and the system expected production capacity $S(t)$. Their behaviours vs time are shown in Figure 7. After about $2500 \mathrm{hr}$ the asymptotic behaviour is reached. Their average values over a period of one year $\left(t_{s}=8760 h r\right)$ are $\bar{A}\left(t_{s}\right)=0.96$, and $\bar{S}\left(t_{s}\right)=88.47 \%$ respectively.

\subsection{Availability sensitivity to one parameter}

To study the system sensitivity using the proposed importance measure MSM, many different directions of sensitivity can be considered. We first consider a directional perturbation matrix $\mathbf{Q}_{\lambda_{i}}$ corresponding to changes in the direction of a single parameter of interest, e.g. the failure rate of component $i, \lambda_{i}$. The corresponding importance measures are denoted $\operatorname{MSM}_{\lambda_{i}}^{A}(t)=\operatorname{MSM}_{\mathbf{Q}_{\lambda_{i}}}^{A}(t)$ for the transient response, $\operatorname{MSM}_{\lambda_{i}}^{\bar{A}}=\operatorname{MSM}_{\mathbf{Q}_{\lambda_{i}}}^{\bar{A}}$ for the average over a finite time horizon and 


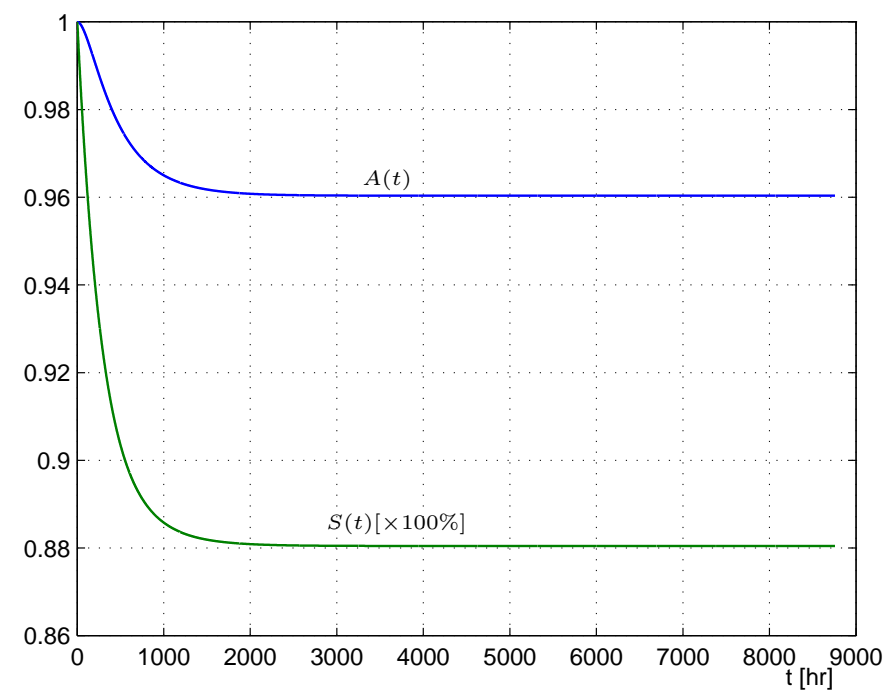

Figure 7: System availability \& expected production capacity.

$\mathrm{MSM}_{\lambda_{i}}^{A}=\operatorname{MSM}_{\mathbf{Q}_{\lambda_{i}}}^{A}$ at steady state. These quantities are evaluated by numerical integration of Equations (10), (14) and (17).
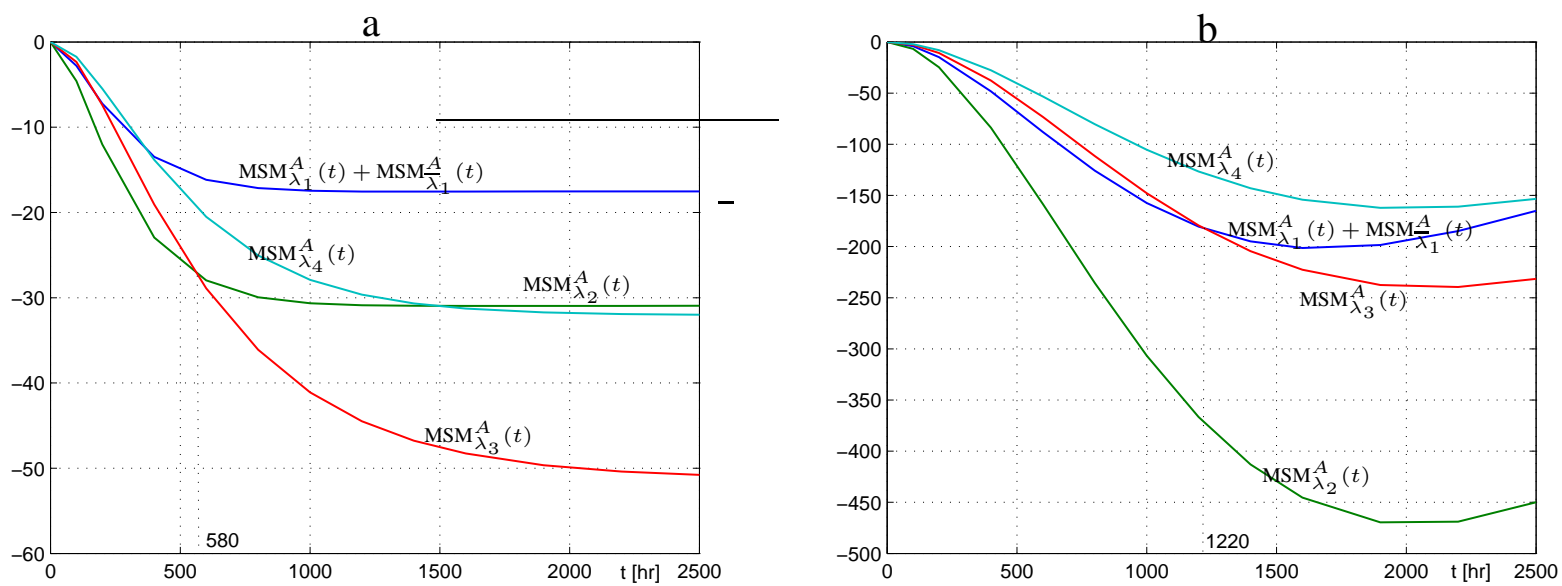

Figure 8: Availability sensitivity to failure rates - (a) with component repairs - (b) without component repair $\left(\mu_{i}=0\right)$

The behavior $v s$ time of the system availability sensitivities with respect to the failure rates are shown in Figure 8, with component repair (8.a) and without component repair (8.b). Obviously, a failure rate increase results in a decrease of the system availability and all MSM values are negative. The sensitivity of the system availability to $C_{1}$ is shared between the sensitivity to $\bar{\lambda}_{1}$ and to $\lambda_{1}$. Hence, because of the linearity in $\mathbf{Q}$ of Equation (10), the impact of failure rate of $C_{1}$ on the system availability sensitivity is given by the $\operatorname{sum}_{\mathrm{MSM}_{\bar{\lambda}_{1}}}^{A}(t)+\operatorname{MSM}_{\lambda_{1}}^{A}(t)$. The analysis of the components sensitivities during the transient state shows that the components importance 
ranking may change with time, see Figure 8.a

- the most important component during a short period of time $(0,580 \mathrm{hr}]$ is $C_{2}$;

- from $t=580 h r$ to $t=1500 h r$, the components importance ranking is $C_{1}<C_{4}<C_{2}<C_{3}$;

- finally, from $t=1500 h r$ until steady state, the components importance ranking is $C_{1}<$ $C_{2}<C_{4}<C_{3}$. This last ranking still holds for the average values during a period of one year presented in Table 3 and also for the availability sensitivity at steady state, in Table 4.

Since the components importance ranking may change with time, the interest of a sensitivity measure during the transient state is precisely to be able to indicate which is the most important component at a given time. Note however that the components' ranking that we obtain are not absolute importance rankings, but the ranking relative to the MSM criterion. Obviously, different rankings could be obtained if a different reliability importance measure was used.

\subsection{Production capacity sensitivity to one parameter}

Consider now the application of MSM for system productivity analysis. As in the previous section, many different directions of interest can be investigated for the production capacity sensitivity study. To illustrate the advantage of MSM in the production capacity analysis, we consider again a directional perturbation matrix $\mathbf{Q}_{\lambda_{i}}$ corresponding to changes in the direction of a single parameter of interest, e.g. the failure rate of component $i, \lambda_{i}$ The corresponding importance measures are denoted $\operatorname{MSM}_{\lambda_{i}}^{S}(t)=\operatorname{MSM}_{\mathbf{Q}_{\lambda_{i}}}^{S}(t)$ for the transient response, $\operatorname{MSM}_{\lambda_{i}}^{\bar{S}}=\operatorname{MSM}_{\mathbf{Q}_{\lambda_{i}}}^{\bar{S}}$ for the average over a finite time horizon and $\operatorname{MSM}_{\lambda_{i}}^{S}=\operatorname{MSM}_{\mathbf{Q}_{\lambda_{i}}}^{S}$ at steady state. These quantities are evaluated by numerical integration of Equations (19), (21) and (22).

Figure 9 sketches the importance measures $\operatorname{MSM}_{\lambda_{i}}^{S}(t)(i=1, \ldots, 4)$ for the cases with component repairs (Figure 9.a) and without component repairs (Figure 9.b). Not surprisingly again, the results show that an increase of a failure rate leads to a decrease of the system productivity. In the case of repairable components, $C_{1}$ turns out to be the most critical component, and the components importance ranking is $C_{4}<C_{3}<C_{2}<C_{1}$. This order remains the same for the average productivity sensitivity during a period of one year, and for the productivity sensitivity at infinite time horizon (see Table 3).

This ranking is not the same as the components importance ranking according to the system availability sensitivity obtained in the previous section since the supply of production for the group $G 2\left(C_{3}\right.$ or $\left.C_{4}\right)$ depends on the state of group $G 1\left(C_{1} \& C_{2}\right)$, hence the impact of $C_{1} \& C_{2}$ on the system production capacity is more important than $C_{3} \& C_{4}$ ("bottleneck effect"). Moreover, the 

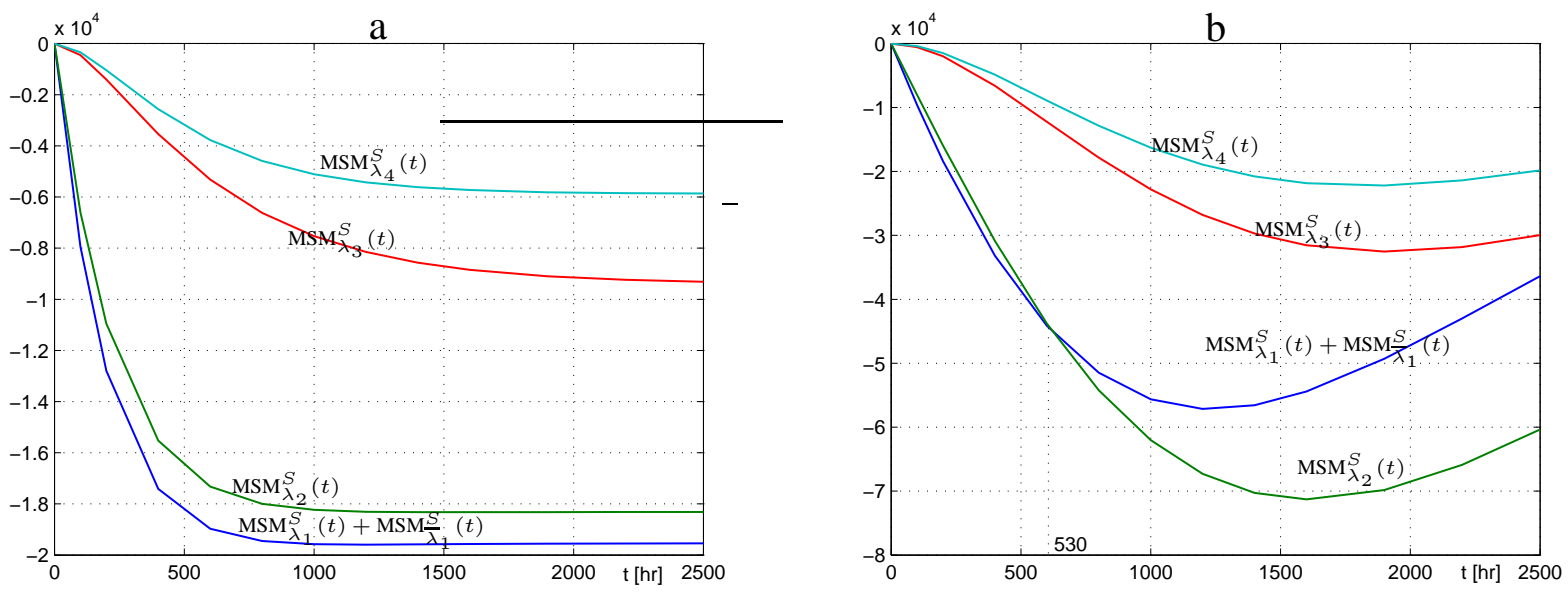

Figure 9: Production capacity sensitivity to failure rates - (a) with component repairs - (b) without component repair $\left(\mu_{i}=0\right)$

production capacity of $C_{1}$ is higher than that of $C_{2}$.

Table 3: Sensitivity to failure rates for a period of one year.

\begin{tabular}{|c|c|c|c|c|c|c|}
\hline \multirow[b]{2}{*}{ Unit } & \multicolumn{3}{|c|}{ Availability } & \multicolumn{3}{|c|}{ Production capacity } \\
\hline & & Value & Order & & Value & Order \\
\hline \multirow{2}{*}{$C_{1}$} & $\operatorname{MSM}_{\lambda_{1}}^{\bar{A}}$ & -7.9066 & \multirow{2}{*}{4} & $\operatorname{MSM}_{\lambda_{1}}^{\bar{S}}$ & -17333.417 & \multirow{2}{*}{1} \\
\hline & $\operatorname{MSM}_{\bar{\lambda}_{1}}^{\bar{A}}$ & -9.0608 & & $\operatorname{MSM} \frac{\bar{S}}{\bar{\lambda}_{1}}$ & -1812.4142 & \\
\hline$C_{2}$ & $\operatorname{MSM}_{\lambda_{2}}^{\bar{A}}$ & -29.8912 & 3 & $\operatorname{MSM}_{\lambda_{2}}^{\bar{S}}$ & -17872.5520 & 2 \\
\hline$C_{3}$ & $\operatorname{MSM}_{\lambda_{3}}^{\bar{A}}$ & -47.3851 & 1 & $\operatorname{MSM}_{\lambda_{3}}^{\bar{S}}$ & -8690.0876 & 3 \\
\hline$C_{4}$ & $\operatorname{MSM}_{\lambda_{4}}^{\bar{A}}$ & -30.0011 & 2 & $\operatorname{MSM}_{\lambda_{4}}^{\bar{S}}$ & -5501.9786 & 4 \\
\hline
\end{tabular}

\subsection{Sensitivity analysis to a group of parameters}

In this section, composite directions of sensitivity are considered, i.e. the perturbation is not limited to a single specific parameter. Instead, perturbations on a group of parameters, or more generally, perturbations on a group of transition rates are studied. The analysis of sensitivity in such directions can help e.g. to identify the importance of group of components or to optimize the maintenance effort.

- Application for groups importance ranking

In Table 5, the direction denoted $\mathbf{Q}\left(\bar{\lambda}_{1}, \lambda_{1}, \lambda_{2}\right)$ indicates that the failure rates of $C_{1}$ and $C_{2}$ are simultaneously perturbed by the same amount. Similarly, the direction denoted 
Table 4: Sensitivity to failure rates at steady state.

\begin{tabular}{|c|c|c|c|c|c|c|}
\hline \multirow[b]{2}{*}{ Unit } & \multicolumn{3}{|c|}{ Availability } & \multicolumn{3}{|c|}{ Production capacity } \\
\hline & & Value & Order & & Value & Order \\
\hline \multirow{2}{*}{$C_{1}$} & $\operatorname{MSM}_{\lambda_{1}}^{A}$ & -8.1398 & \multirow{2}{*}{4} & $\operatorname{MSM}_{\lambda_{1}}^{S}$ & -17644.2201 & \multirow{2}{*}{1} \\
\hline & $\operatorname{MSM}_{\bar{\lambda}_{1}}^{A}$ & -9.3909 & & $\operatorname{MSM}{\frac{S}{\lambda_{1}}}$ & -1897.74279 & \\
\hline$C_{2}$ & $\operatorname{MSM}_{\lambda_{2}}^{A}$ & -30.9503 & 3 & $\operatorname{MSM}_{\lambda_{2}}^{S}$ & -18310.9108 & 2 \\
\hline$C_{3}$ & $\operatorname{MSM}_{\lambda_{3}}^{A}$ & -51.2382 & 1 & $\operatorname{MSM}_{\lambda_{3}}^{S}$ & -9395.1702 & 3 \\
\hline$C_{4}$ & $\operatorname{MSM}_{\lambda_{4}}^{A}$ & -32.0239 & 2 & $\operatorname{MSM}_{\lambda_{4}}^{S}$ & -5871.9814 & 4 \\
\hline
\end{tabular}

Table 5: Sensitivity analysis to group of parameters

\begin{tabular}{ccccc}
\hline & \multicolumn{2}{c}{ Availability } & \multicolumn{2}{c}{ Production capacity } \\
\cline { 2 - 5 } Direction & $\mathrm{MSM}_{\mathbf{Q}}^{\bar{A}}$ & $\mathrm{MSM}_{\mathbf{Q}}^{A}$ & $\mathrm{MSM}_{\mathbf{Q}}^{\bar{S}}$ & $\mathrm{MSM}_{\mathbf{Q}}^{S}$ \\
\hline$Q\left(\bar{\lambda}_{1}, \lambda_{1}, \lambda_{2}\right)$ & -46.8586 & -48.4811 & -37018.3834 & -37852.8737 \\
$Q\left(\lambda_{3}, \lambda_{4}\right)$ & -77.3862 & -83.2621 & -14192.0662 & -15267.1516 \\
$Q\left(\bar{\lambda}_{1}, 2.4 \mu_{2}\right)$ & 0.0131 & 0.2304 & 2873.4024 & 3050.3997 \\
$Q\left(\lambda_{4}, 2.53 \mu_{3}\right)$ & -0.0340 & 1.5115 & -6.1964 & 277.1575 \\
\hline
\end{tabular}

$\mathbf{Q}\left(\lambda_{3}, \lambda_{4}\right)$ corresponds to the simultaneous perturbation of the failure rates of $C_{3}$ and $C_{4}$ by the same amount. Hence, the derivative of the system performance in one of these directions gives an importance measure of the effect of the corresponding group of components on the system performance. According to these measures on the system availability during a period of one year and at steady state, the groups'/components' ranking is $C_{1}<C_{2}<C_{4}<G 1\left(C_{1}, C_{2}\right)<C_{3}<G 2\left(C_{3}, C_{4}\right)$. The groups/components importance measures with respect to the system productivity can also be derived and results in the following ranking $C_{4}<C_{3}<G 1\left(C_{3}, C_{4}\right)<C_{2}<C_{1}<G 2\left(C_{1}, C_{2}\right)$.

- Application to maintenance optimisation When one parameter of the system is changed (increased failure rate, components degradation, for example), the system performances (availability, productivity) deteriorates. This variation can be compensated completely or partially if at the same time, other parameters of the systems (repair rates, for example) can be perturbed to compensate for this change in performance. This action can be performed by choosing a suitable direction of perturbation $\mathbf{Q}$. In Table 5, two directions of perturbation are proposed to keep the system availability or/and the system productivity at the same level in the case of a degradation components $C_{1}$ and $C_{4}$. The direction $\mathbf{Q}\left(\lambda_{i}, \alpha \mu_{j}\right)$ indicates that if $\lambda_{i}$ (for $i=1,4$ ) is increased by an amount $\delta$, then at the same time, $\mu_{j}$ is perturbed by 
Table 6: Sensitivity analysis to the failure rate of a given state

\begin{tabular}{cccc|ccc}
\hline & \multicolumn{3}{c|}{ Availability } & \multicolumn{3}{c}{ Production capacity } \\
\cline { 2 - 7 } Direction & $\mathrm{MSM}_{\mathbf{Q}_{S i}}^{\bar{A}}$ & $\mathrm{MSM}_{\mathbf{Q}_{S i}}^{A}$ & Order & $\operatorname{MSM}_{\mathbf{Q}_{S i}}^{\bar{S}}$ & $\mathrm{MSM}_{\mathbf{Q}_{S i}}^{S}$ & Order \\
\hline$Q_{S 1}$ & -57.4255 & -60.0535 & 1 & -35477.8421 & -36215.8937 & 1 \\
$Q_{S 2}$ & -13.8533 & -14.6393 & 3 & -2354.4050 & -2512.5380 & 3 \\
$Q_{S 3}$ & -11.4106 & -12.0197 & 4 & -2221.8456 & -2356.8238 & 4 \\
$Q_{S 4}$ & -27.3454 & -29.1022 & 2 & -8022.0121 & -8486.7967 & 2 \\
$Q_{S 5}$ & -5.1681 & -6.0478 & 5 & -1496.7651 & -1739.0416 & 5 \\
$Q_{S 7}$ & -4.0821 & -4.4008 & 6 & -719.7299 & -785.4732 & 6 \\
$Q_{S 8}$ & -0.7728 & -0.9072 & 8 & -136.2435 & -162.0568 & 8 \\
$Q_{S 9}$ & -3.5206 & -3.7906 & 7 & -657.3550 & -714.1543 & 7 \\
$Q_{S 10}$ & -0.6664 & -0.7822 & 9 & -124.2511 & -147.2472 & 9 \\
\hline
\end{tabular}

an amount $\alpha \delta$. A sensitivity close to zero in a direction of the form $\mathbf{Q}\left(\lambda_{i}, \alpha \mu_{j}\right)$ indicates that the change on $\mu_{j}$ almost balances the effect of the change on $\lambda_{i}$. From a practical point of view, this can be seen as a mean to tune the maintenance parameters, such that a perturbation on the failure rate has no impact on the system availability or/and system productivity. Maintenance policies parameters can then be optimally tuned in this way and the optimal solution can also depend on other criteria (maintenance cost, for example).

\subsection{Sensitivity analysis to failure rates of a given state}

To study the sensitivity of a given state, some specific directions of sensitivity are considered. In Table 6, the direction denoted $\mathbf{Q}_{S i}$ indicates that all failure rate transitions out from the operational state $i(i=1,2,3,4,5,7,8,9,10)$ are simultaneously perturbed by the same amount. The sensitivity in these directions can help to identify the importance of a given state. According to the sensitivity in these directions on the system availability and/or on the system productivity during a period of one year and at steady state, the most important state is state number 1 and state 10 is the least important one, see Table 6. As shown in Figure 10.a (sensitivity of the system availability for different states $S_{i}$ ) and in Figure 10.b (sensitivity of the system productivity), during the transient state the states importance ranking can change with time.

\subsection{Sensitivity analysis to failure rates of a group of states}

This subsection explores the sensitivity of the failure rates of a group of states. In Table 7, the direction denoted $\mathbf{Q}_{\left(C_{i} C_{j}\right)}(i, j=1,2,3,4)$ indicates that the failure rates of components $C_{i}$ and $C_{j}$ are simultaneously perturbed by the same amount in all the operational states where both 

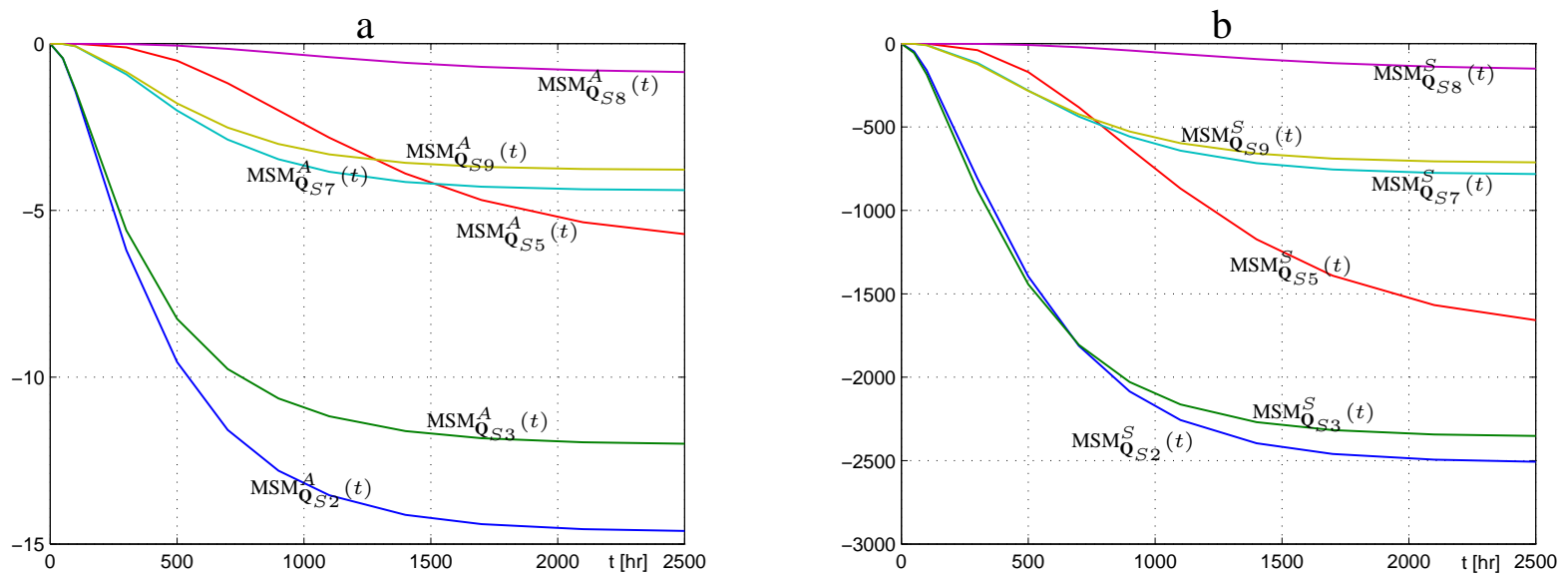

Figure 10: Sensitivity to failure rates of a given state - (a) Sensitivity of the system availability - (b) Sensitivity of the system production capacity

$C_{i}, C_{j}$ are functioning. From a practical point of view, this perturbation could be caused by, for example, electrical shock, environmental conditions changing, etc. The sensitivity of the system availability/productivity in this direction allows for the quantification of the impact of a group of operational components on the system availability/productivity during a period of one year and at steady state, see Table 7

- according to the sensitivity on the system availability, the most important group of 2 operational components is $\left(C_{2}, C_{3}\right)$ and the group $\left(C_{1}, C_{2}\right)$ is the least important one;

- according to the sensitivity on the system productivity, the most important group of 2 operational components is $\left(C_{1}, C_{2}\right)$ and the group $\left(C_{2}, C_{4}\right)$ is the least important one.

The results for the transient state are given in Figure 11.a for the system availability sensitivity analysis and in Figure 11.b for the system productivity sensitivity analysis.

Table 7: Sensitivity analysis to the failure rates of a group of states

\begin{tabular}{ccccc|ccc}
\hline & & \multicolumn{2}{c|}{ Availability } & \multicolumn{3}{c}{ Production capacity } \\
\cline { 3 - 8 } Direction & States & MSM $_{\mathbf{Q}}^{\bar{A}}$ & MSM $_{\mathbf{Q}}^{A}$ & Order & MSM $_{\mathbf{Q}}^{\bar{S}}$ & MSM $_{\mathbf{Q}}^{S}$ & Order \\
\hline$Q_{\left(C_{1} C_{2}\right)}$ & $1,4,5$ & -26.4276 & -27.2344 & 5 & -33329.7076 & -33978.7511 & 1 \\
$Q_{\left(C_{1} C_{3}\right)}$ & $1,3,5,10$ & -58.0905 & -61.8841 & 2 & -25005.6896 & -25899.9678 & 2 \\
$Q_{\left(C_{1} C_{4}\right)}$ & 4,9 & -28.9252 & -30.8173 & 4 & -6998.0912 & -7425.4585 & 4 \\
$Q_{\left(C_{2} C_{3}\right)}$ & $1,2,5,8$ & -70.1417 & -74.3927 & 1 & -23954.6266 & -24903.1600 & 3 \\
$Q_{\left(C_{2} C_{4}\right)}$ & 4,7 & -30.5990 & -32.6183 & 3 & -6918.9540 & -7354.4199 & 5 \\
\hline
\end{tabular}


$\mathrm{a}$

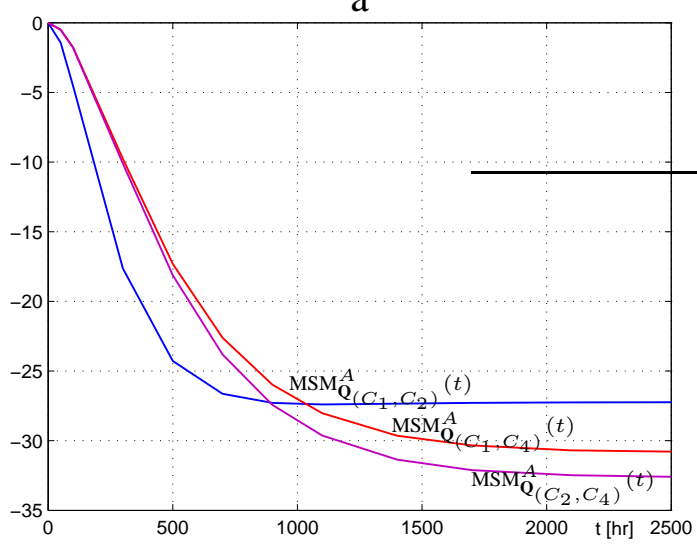

$\mathrm{b}$

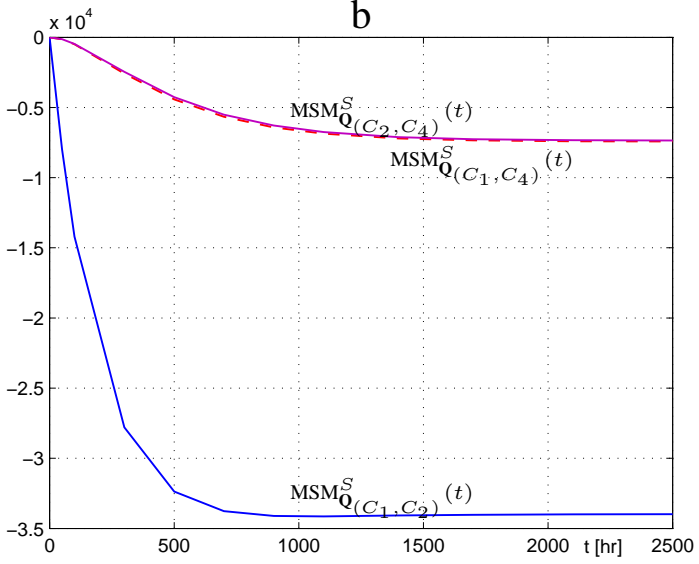

Figure 11: Sensitivity to failure rates of a group of states - (a) Sensitivity of the system availability - (b) Sensitivity of the system production capacity

\section{Conclusions}

The multi-directional sensitivity measure, MSM, can be used to investigate the performance sensitivity of dynamic systems in any direction of one parameter, or in any direction of a group of parameters, and, more generally, the effect of the simultaneous change of several design parameters. This measure can be extended to the multi-state production systems whose performance output is not only the system availability but also its production capacity. The sensitivity of both performance outputs are studied in the transient state, during a given period of time and at steady state. On the basis of the results of the sensitivity analysis in the different directions of interest, the most critical component or the group of most critical components can be identified. The maintenance policies parameters can be also tuned to keep a constant availability and/or productivity level in the presence of components degradation, etc...

This paper is the development of our research in the framework of the sensitivity importance analysis of dynamic systems presented in part in [3]. Our future research work focuses on the direction sensitivity optimization for maintenance policy parameters, and on the development of methods to estimate our proposed measure MSM with the operating feedback data in the transient state.

\section{References}

[1] A.Gandini. Importance and sensitivity analysis in assessing system reliability. IEEE Transaction on Reliability, 39(1):61-70, 1990. 
[2] X.-R. Cao and H.-F. Chen. Pertubation realization, potentials, and sensitivity analysis of markov processes. IEEE Transactions on Automatic Control, 42(10):1382-1393, 1997.

[3] P. Do Van, A. Barros, and C. Berenguer. Importance measure on finite time horizon and application to markovian multi-state production systems. In Terje Aven \& Jan Erik Vinnem, editor, Risk, Reliability and Societal Safety - Proc.ESREL 2007, 25-27 June 2007, Stavanger, Norway, Taylor \& Francis Group, pages 229-237. Taylor \& Francis, 2007.

[4] P. Do Van, A. Barros, and C. Berenguer. Reliability importance analysis of markovian systems at steady state using perturbation analysis. Reliability Engineering and System Safety, submitted, 2007.

[5] P. Do Van, S. Khalouli, A. Barros, and C. Berenguer. Sensitivity \& importance analysis of markov models using perturbation analysis: Applications in reliability. In C. Guedes-Soares and E. Zio, editors, Safety and Reliability for Managing Risk - Proc. of ESREL 2006, 18-22 sep. 2006, Estoril, Portugal, pages 1769-1775. Taylor \& Francis, 2006.

[6] P. Frank. Introduction to system sensitivity. Academic press, 1978.

[7] B.H. Hubbard, J.H.and West. Differential Equations: A Dynamical Systems Approach. Texts in Applied Mathematics 18. Springer-Verlag, 1990.

[8] F. Innal and Y. Dutuit. Evaluation de la performance d'un système de production et des contributions individuelles de ses unités constitutives. In Gème Conférence Francophone de Modélisation et Simulation - MOSIM'06, Maroc, 3-5 avril, 2006. In French.

[9] Y. Kawauchi and M. Rausand. A new approach to production regularity assessment in the oil and chemical industries. Reliability Engineering and System Safety, 75:379-388, 2002.

[10] G. Levitin and A. Lisnianski. Importance and sensitivity analysis of multi-state systems using the universal generating function method. Reliability Engineering and System Safety, 65:271-281, 1999.

[11] Jr. Meyer, C.D. The role of the group generalized inverse in the theory of finite markov chains. SIAM Rev., 17:443-464, 1975.

[12] M.F. Neuts. Algorithm Probability: a Collection of Problems. Chapman and Hall, 1995.

[13] Y. Ou and J. Bechta-Dugan. Approximate sensitivity analysis for acyclic Markov reliability models. IEEE Transactions on Reliability, 52(2):220-231, 2003.

[14] Jose E. Ramirez-Marquez and David W. Coit. Composite importance measures for multistate systems with multi-state components. IEEE Transactions on Reliability, 54(3):517$529,2005$. 
[15] M. Rausand and H. Hoyland. System Reliability Theory - Models, Statistical methods and Application. Wiley Series in Probability and Statistics. Wiley Interscience, second edition edition, 2004.

[16] E. Zio and L. Podofillini. Importance measures of multi-state components in multi-state systems. International Journal of Reliability, Quality and Safety Engineering, 10(3):289310, 2003. 